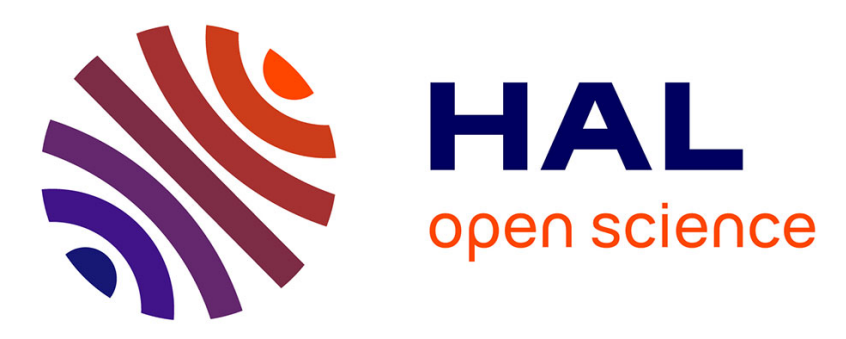

\title{
The three-dimensional transition in the flow around a rotating cylinder
}

Rajah El Akoury, Marianna Braza, Rodolphe Perrin, Gilles Harran, Yannis Hoarau

\section{- To cite this version:}

Rajah El Akoury, Marianna Braza, Rodolphe Perrin, Gilles Harran, Yannis Hoarau. The threedimensional transition in the flow around a rotating cylinder. Journal of Fluid Mechanics, 2008, 607, pp.1-11. 10.1017/S0022112008001390 . hal-02545291

\section{HAL Id: hal-02545291 https://hal.science/hal-02545291}

Submitted on 16 Apr 2020

HAL is a multi-disciplinary open access archive for the deposit and dissemination of scientific research documents, whether they are published or not. The documents may come from teaching and research institutions in France or abroad, or from public or private research centers.
L'archive ouverte pluridisciplinaire HAL, est destinée au dépôt et à la diffusion de documents scientifiques de niveau recherche, publiés ou non, émanant des établissements d'enseignement et de recherche français ou étrangers, des laboratoires publics ou privés. 


\title{
The three-dimensional transition in the flow around a rotating cylinder
}

\author{
R. EL AKOURY ${ }^{1}, M$. BRAZA ${ }^{1}, R$. PERRIN ${ }^{1} \dagger, G . H_{A R R A N^{1}}$ \\ AND Y. HOARA U ${ }^{2}$ \\ ${ }^{1}$ Institut de Mécanique des Fluides de Toulouse, CNRS/INPT/UPS UMR 5502, \\ Toulouse, France \\ ${ }^{2}$ Institut de Mécanique des Fluides et des Solides de Strasbourg,CNRS/ULP UMR 7507, \\ Strasbourg, France
}

(Received 20 September 2007 and in revised form 21 February 2008)

\begin{abstract}
The flow around a circular cylinder rotating with a constant angular velocity, placed in a uniform stream, is investigated by means of two- and three-dimensional direct numerical simulations. The successive changes in the flow pattern are studied as a function of the rotation rate. Suppression of vortex shedding occurs as the rotation rate increases $(>2)$. A second kind of instabilty appears for higher rotation speed where a series of counter-clockwise vortices is shed in the upper shear layer. Threedimensional computations are carried out to analyse the three-dimensional transition under the effect of rotation for low rotation rates. The rotation attenuates the secondary instability and increases the critical Reynolds number for the appearance of this instability. The linear and nonlinear parts of the three-dimensional transition have been quantified by means of the amplitude evolution versus time, using the Landau global oscillator model. Proper orthogonal decomposition of the three-dimensional fields allowed identification of the most energetic modes and three-dimensional flow reconstruction involving a reduced number of modes.
\end{abstract}

\section{Introduction}

The flow around a circular cylinder is representative of many phenomena that occur in fluid dynamics. This study describes the transition to turbulence in the wake past a rotating circular cylinder, by means of two- and three-dimensional direct numerical simulation. There are a number of experimental investigations and of DNS studies concerning the three-dimensional vortex dynamics of a fixed cylinder, e.g. Williamson (1992), Persillon \& Braza (1998), and Braza, Faghani \& Persillon (2001), but only a few numerical studies devoted to the three-dimensional transition including wall rotation effects. The flow field depends mainly on two parameters, the Reynolds number $R e$ and the rotation rate $\alpha: \operatorname{Re}=U_{\infty} D / v$ where $D$ is the cylinder diameter, $U_{\infty}$ is the free-stream velocity and $v$ is the kinematic viscosity of the fluid; $\alpha=D \omega_{0} /\left(2 U_{\infty}\right)$ where $\omega_{0}$ is the angular velocity of the cylinder.

The earliest experiments on the flow past a circular rotating cylinder were performed by Prandtl (1925). More recently, the early phase of the establishment of the flow past a circular cylinder started impulsively into rotation and translation was investigated

$\dagger$ Present address: Laboratoire d'Etudes Aérodynamiques, CNRS/Univ. Poitiers/ENSMA UMR $\mathrm{N}^{\circ} 6609$, France 


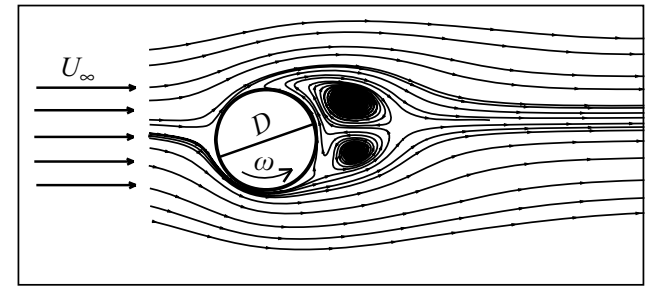

FIGURE 1. Schematic of the physical problem.

experimentally and numerically by Badr et al. (1990). They observed that there is no periodic vortex shedding for $\alpha>2$. Concerning the established state, the twodimensional flow for low and moderate Reynolds number under the rotation effect was studied by Ingham \& Tang (1990) and later by Kang, Choi \& Lee (1999). Stojkovic, Breuer \& Durst (2002) were the first to notice the existence of a second shedding mode for $4.8 \leqslant \alpha \leqslant 5.15$ at $R e=100$. The two-dimensional numerical study of Stojkovic et al. (2003) confirmed the existence of this second mode in the Reynolds number range $60 \leqslant R e \leqslant 200$. Different flow regimes as rotation speed increases were investigated by Mittal \& Kumar (2003), at $R e=200,0 \leqslant \alpha \leqslant 5$. Cliffe \& Tavener (2004) studied the effect of the rotation of a cylinder on the critical Reynolds and Strouhal numbers at the Hopf bifurcation point. Apart from the study of Tokumaru $\&$ Dimotakis (1993) on the aspect ratio influence on the mean lift coefficient, and the more recent study of Mittal (2004) $(R e=200$ and $\alpha=5)$, to our knowledge there are no studies qualifiying the three-dimensional effects of the rotation in the Reynolds number range of the present study.

In the present paper, the flow around a rotating circular cylinder placed in a uniform stream, figure 1 , is investigated by means of two- and three-dimensional direct numerical simulations, using the code ICARE/IMFT, Braza, Chassaing \& Ha-Minh (1986), Persillon \& Braza (1998). The objectives of the present work are summarized as follows:

(a) To analyse the onset of the three-dimensional transition phenomena under the rotation effect by means of direct numerical simulations, which offers the possibility of dissociating the purely three-dimensional from the two-dimensional mechanisms, which co-exist and interact in the physical experiment.

(b) To study the amplification of the three-dimensional instability process in the spanwise direction using the DNS results, by the Landau global oscillator model, and find the critical Reynolds number for the appearance of the secondary instablity under the rotation effect.

(c) To analyse the organized modes under the rotation effect by a three-dimensional proper orthogonal decomposition.

\section{Summary of the numerical method}

The two- and three-dimensional simulations were carried out using the code ICARE of the IMFT, in finite-volume formulation. The governing equations are the continuity and the Navier-Stokes equations for an incompressible fluid, written in general curvilinear coordinates in the $(x, y)$-plane, while the spanwise component $z$ is in Cartesian coordinates. The numerical method is based on the pressurevelocity formulation using a predictor-corrector pressure scheme, Braza et al. (1986). The temporal discretization adopts the Peaceman \& Rachford (1955) scheme in an 
alternating-direction-implicit formulation. The method is second-order accurate in time and space. The staggered grids by Harlow \& Welch (1965) are employed for the velocity and pressure variables.

H-type grids are used because they allows more physical boundary conditions to be introduced on the external boundaries avoiding branch-cut lines. A grid dependence study for the three-dimensional flow around a cylinder with the same code is presented in Persillon \& Braza (1998), and optimum grids have been chosen in the present study. The grid used is $(250 \times 100 \times 80)$. The spanwise length of the computational domain is $12 D$ where $D$ is the cylinder diameter. The boundary conditions include a nonreflecting condition at the outer boundary to avoid feedback effects, according to Jin \& Braza (1993). A detailed presentation of the boundary conditions for the cylinder can be found in Persillon \& Braza (1998). For the spanwise free edges of the computational domain, periodic boundary conditions are applied. All presented physical quantities are made dimensionless using upstream velocity and cylinder diameter.

\section{Successive stages of the two-dimensional transition}

For low Reynolds numbers, $R e<48$, the flow remains steady as the rotation rate increases. For higher Reynolds numbers, an increase in the rotation rate yields successive changes to the flow regime, as described below. At each Reynolds number, the same successive changes occur as a function of $\alpha$. For low rotation rate $\alpha<\alpha_{L 1}$, figure 2 shows unsteady flow development similar to the von-Kármán vortex shedding (mode I). This pattern is asymmetric towards the upper part of the cylinder due to the rotation sense. The effect of increasing rotation is to attenuate the contra-rotating vortices from the lower side of the cylinder; thus the instability mode vanishes and the flow is steady for $\alpha_{L 1}<\alpha<\alpha_{L 2}$. Owing to the increasing rotation rate in association with the strong viscous effect near the wall, the shearing mechanism is increased, and a second instability, mode II, appears in the range $\alpha_{L 2}<\alpha<\alpha_{L 3}$. In this interval, only counter-clockwise vortices are shed from the upper part of the cylinder with a large wavelength (figure 2, bottom). For $\alpha>\alpha_{L 3}$, the shearing rate becomes very high and increases the viscous effects near the wall. As a result, the flow becomes steady. For $\alpha<\alpha_{L 1}$, the Strouhal number $S t=f D / U_{\infty}$ of vortex shedding is practically constant and decreases as a function of $\alpha$ before the first bifurcation, and is very low in the second mode interval, as shown in figure $3(b)$. The critical $\alpha$ values are presented in figure 3( $a$ ) for different Reynolds numbers in comparison with results of Mittal \& Kumar (2003) and Stojkovic et al. (2002, 2003). This figure shows that the critical Reynolds number for the appearance of the first flow unsteadiness increases with $\alpha$.

\section{Three-dimensional transition}

The objectives of this section are to analyse the onset and modification of three-dimensional transition phenomena under the rotation effect with respect to the coherent structures in the wake. Without rotation, at $R e=200$, the threedimensionality starts from an amplification of the $w$-velocity component (in the spanwise direction) versus time in the near wake (figure $4 a$ ). There is a linear amplification rate followed by a nonlinear state that leads finally to a saturation state as reported by Persillon \& Braza (1998). The $w$ amplification corresponds to the development of a secondary instability involving the three-dimensional modification of the von Kármán mode which starts to display a regular spanwise undulation 

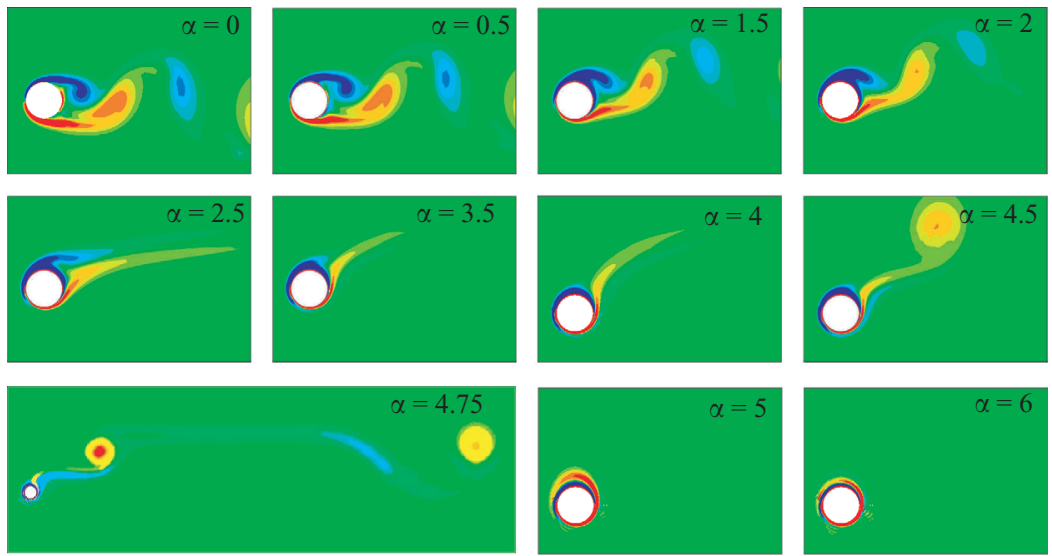

$\omega_{z}:-2.000-1.111-0.2220 .2221 .1112 .000$

FIGURE 2. Iso-vorticity $-2 \leqslant \omega_{z} \leqslant+2$, successive steps in the two-dimensional transition as $\alpha$ increases, $R e=200$.
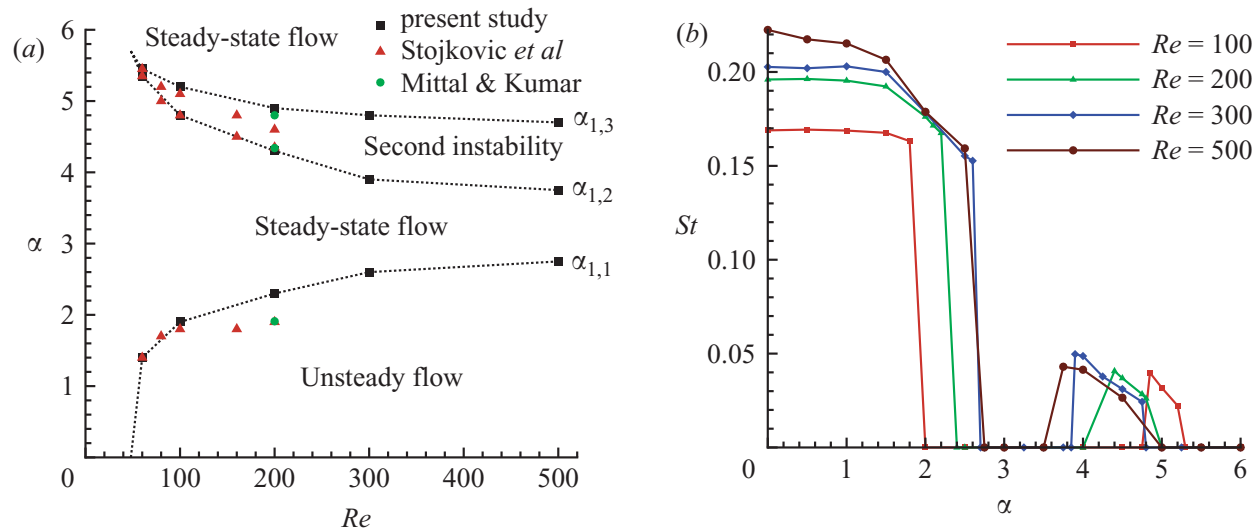

FIGURE 3. Global parameters of the two-dimensional transition: (a) critical rotation rate versus Reynolds number, stability diagram; (b) St as a function of $\alpha$ for different $R e$.

(mode A). The spanwise undulation is shown in figure 5(a)(i), where the spanwise wavelength $\lambda_{z} / D$ is found equal to 4 . When wall rotation is applied $(\alpha=1.5)$ the flow displays a drastic damping of the $w$ amplitudes. Three different initial conditions were used: a three-dimensional field from DNS at $\operatorname{Re}=200$ and $\alpha=0$ (figure $4 a$ ): flow at rest, $R e=200$ and $\alpha=1.5$; and a two-dimensional field at the same $R e$ and $\alpha$. The same kind of $w$ damping was found for all three different initial conditions. Therefore there is no secondary instability for $R e=200$ and $\alpha=1.5$, and the rotation increases the critical Reynolds number for the appearance of this instability. At higher Reynolds number, $R e=300$, the amplification of the $w$ component is clearly shown (figure $4 c$ ) for $\alpha=0.5$, the initial condition being from a two-dimensional NavierStokes simulation with $\alpha=0.5$. The established state is shown in figure 5(a)(iii), where the spanwise undulation is seen, with $\lambda_{z} / D=4$, figure $5(b)$, showing that the rotation effects maintain mode $\mathrm{A}$ even at $R e=300$. 
Three-dimensional transition in the flow around a rotating cylinder
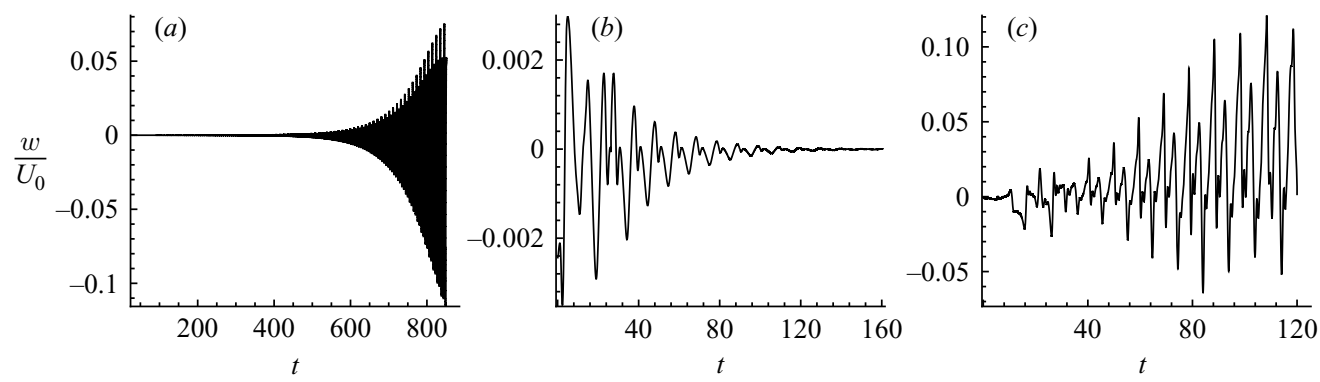

FIGURE 4. Temporal evolution of the $w$-velocity component at $(x / D, y / D, z / D)=(1.47,0,6)$ (a) $\operatorname{Re}=200, \alpha=0$; (b) $\operatorname{Re}=200, \alpha=1.5$; (c) $\operatorname{Re}=300, \alpha=0.5$.
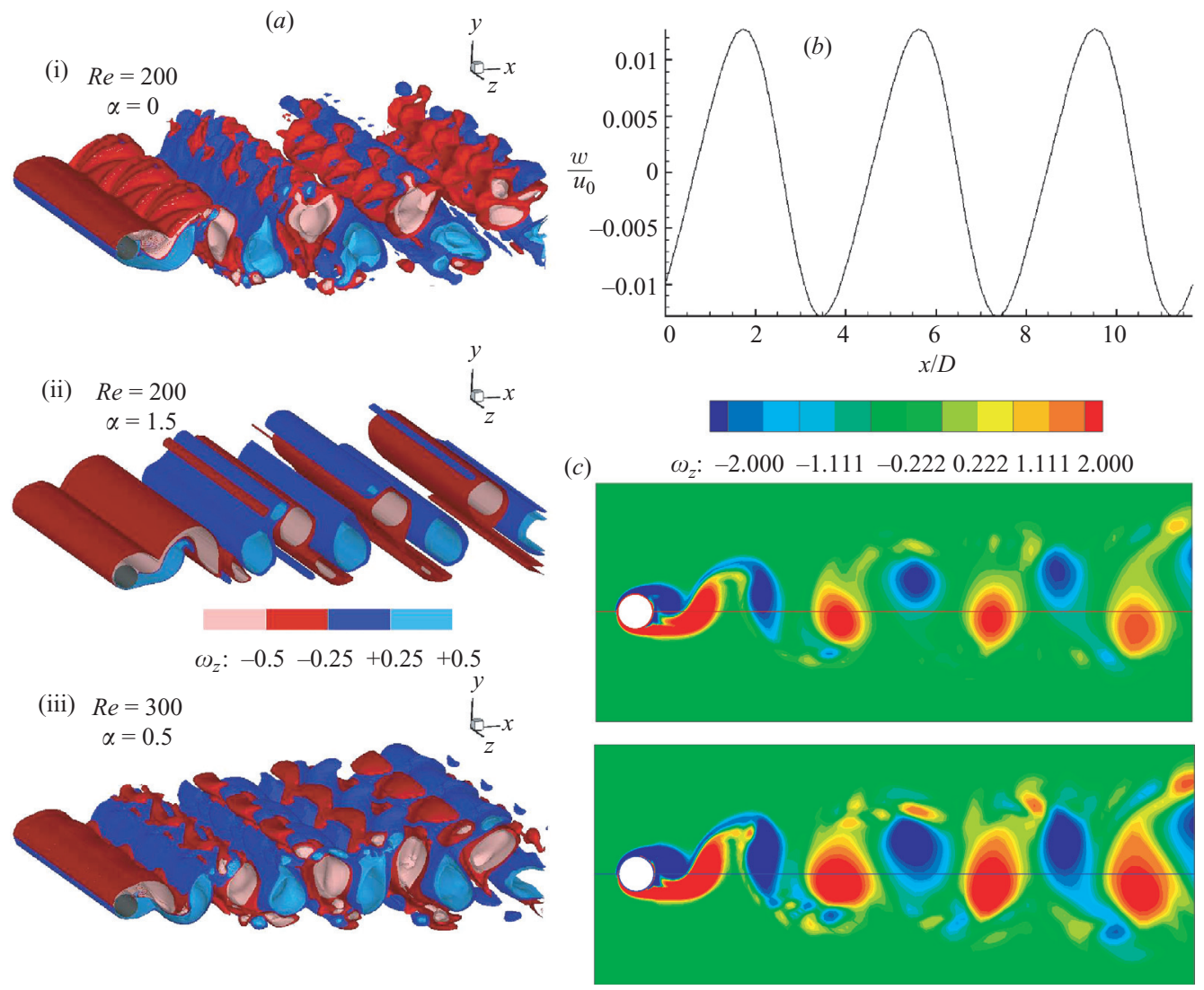

FiguRE 5. Secondary instability and spanwise evolution of the coherent vorticies. $(a)$ Iso-vorticity surfaces for three-dimensional fields, $\omega_{z}= \pm 0.25, \pm 0.5$. (b) Spanwise undulation of $w / U_{0}, R e=300, \alpha=0.5 ;(x / D, y / D)=(1.01,1.5)$. (c) $\omega_{z}$ iso-vorticity contours; top, two-dimensional simulations; bottom, three-dimensional simulations, $R e=300, \alpha=0.5$.

The median section of the flow is compared for a two- and three-dimensional computation, $z / D=6$, (figure $5 c$ ) to split the purely three-dimensional effects from the two-dimensional ones. A number of smaller-scale vortices in the shear layer appear in the three-dimensional simulations. The von Kármán vortices are larger in 

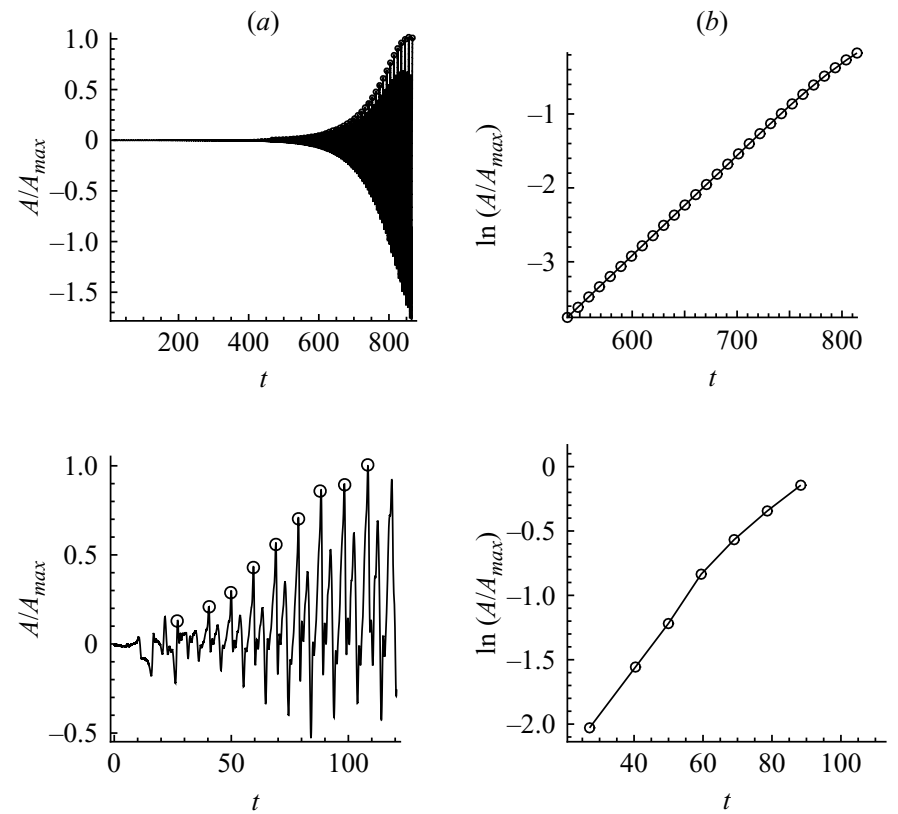

FIgURE 6. (a) Amplification of the $w$-velocity component; $(b)$ normalized logarithmic amplitude evolution versus time, evaluation of $\sigma_{r}$. Top $R e=200, \alpha=0$; bottom $R e=300, \alpha=0.5$.

the three-dimensional case. The rotation in three-dimensional computations induces a modification in the strain axis of the von Kármán vortices.

The amplification of the secondary instability under the wall rotation effect can be studied by means of the Landau global oscillator model, Provansal, Mathis \& Boyer (1987),

$$
\frac{\partial A}{\partial t}=\underbrace{\sigma_{r} A}_{\text {linear growth }}-\underbrace{l_{r}\left|A^{3}\right|}_{\text {nonlinear }} .
$$

The real parts of the coefficients $\sigma_{r}$ and $l_{r}$ can be evaluated by the present DNS study, providing the amplitude variation as a function of period, $A / A_{\text {max }}$, figure $6(a)$. $\sigma_{r}$ can be evaluated by the $\log \left(A / A_{\max }\right)$ variation with time, figure $6(b)$. For $\operatorname{Re}=200$ $(\alpha=0), \sigma_{r}=0.013$ and for $\operatorname{Re}=300(\alpha=0.5), \sigma_{r}=0.041$. The $\sigma_{r}$ evaluation also allows the assesment of the nonlinear growth coefficient near the saturation threshold $l_{r}=\sigma_{r} \times A /\left|A^{3}\right| . l_{r}$ is found to be positive, which corresponds to a supercritical bifurcation. This means that the bifurcated state exists for Reynolds numbers above critical. $l_{r}=3.055$ and 2.314 for $R e=200$ and 300 respectively.

From the Landau equation (4.1), the critical Reynolds number for the appearance of the secondary instability, for $\alpha=0.5$, can be evaluated by performing threedimensional simulations near the threshold, with $\left(R e-R e_{c r}\right) / R e_{c r}$ a small parameter. The exponential growth coefficient $\sigma_{r}$ can be written as a function of the critical Reynolds number near the threshold: $\sigma_{r}=k\left(R e-R e_{c r}\right)$. According to Provansal et al. (1987), $k$ is a reduced frequency and $\sigma_{r}$ can be also written as $\sigma_{r}=(1 / 5)\left(v / D^{2}\right)\left(R e-R e_{c r}\right)$ where $v$ is the kinematic viscosity and $D$ the cylinder diameter.

If this global oscillator model is verified by the physics of the present bifurcation, $\sigma_{r}$ must therefore vary linearly as a function of Reynolds number near the threshold. 


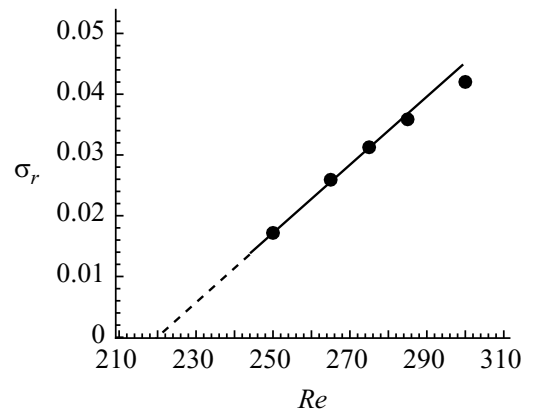

FIGURE 7. Linear amplification versus Reynolds number; assessment of the critical Reynolds number associated to the secondary instability at $\alpha=0.5$.

This is examined by the present direct simulation, for $\alpha=0.5$. Figure 7 shows the $\sigma_{r}$ variation versus Reynolds number. The Reynolds number 300 is too far from the threshold and this point is not taken into account for the assessment of the critical value. The four points corresponding to lower Reynolds numbers clearly indicate a straight line definition that cuts the $R e$ axis at the value 219.8. Therefore, the critical Reynolds number is higher than for the $\alpha=0$ case, evaluated by the DNS of Persillon \& Braza (1998).

The above discussion provides the amplification characteristics of the threedimensional global instability by a DNS approach and by a simpler oscillator model. In the following section we analyse the energy of the organized modes in space and reconstruct the pattern of the three-dimensional coherent structures.

\section{POD analysis}

The analysis of the three-dimensional coherent pattern is carried out by means of a proper orthogonal decomposition (POD), using the snapshot method, Berkooz, Holmes \& Lumley (1993). A subdomain of $160 \times 52 \times 80$ points has been stored for 6 fundamental periods of the votrex shedding; 170 successive instantaneous fields were used. The domain size and time-dependent storage are sufficient to capture the secondary instability and of the main vortex pattern.

Figure $8(a)$ shows the energy diagram of the first 100 POD modes on a log-log scale, and figure $8(b)$ shows a zoom of the first 20 modes on a linear-log scale for Reynolds number 200 at $\alpha=0$ and $\alpha=1.5$. The rotation is found to increase the energy of the modes, as well as to slightly delay the energy decay, for small $\alpha$ values and for two similar Reynolds numbers. Comparing the slope of the energy decay in two-dimensional simulations, figure $8(c)$, and the three-dimensional decay, it can be seen that the latter is less abrupt than in the two-dimensional case. It displays a more chaotic character which is captured by the DNS. Figure 9 shows the topology of the first five POD modes for $R e=300$ and $\alpha=0.5$. Mode 1 is very close to the time-averaged flow, forming two main vortex lobes downstream of the cylinder. The averaged vortex pattern is subjected to a transverse undulation, according to the secondary instability. The two next POD modes, 2 and 3, have comparable energy. They are composed of a single row of opposite-sign patterns. Their three-dimensional evolution shows the insertion of a half-wavelength pattern between the principal undulation shown for mode 1. This pattern is more pronounced in mode 3. Modes 4 and 5 are more fragmented by this half-wavelength spanwise pattern, which is 

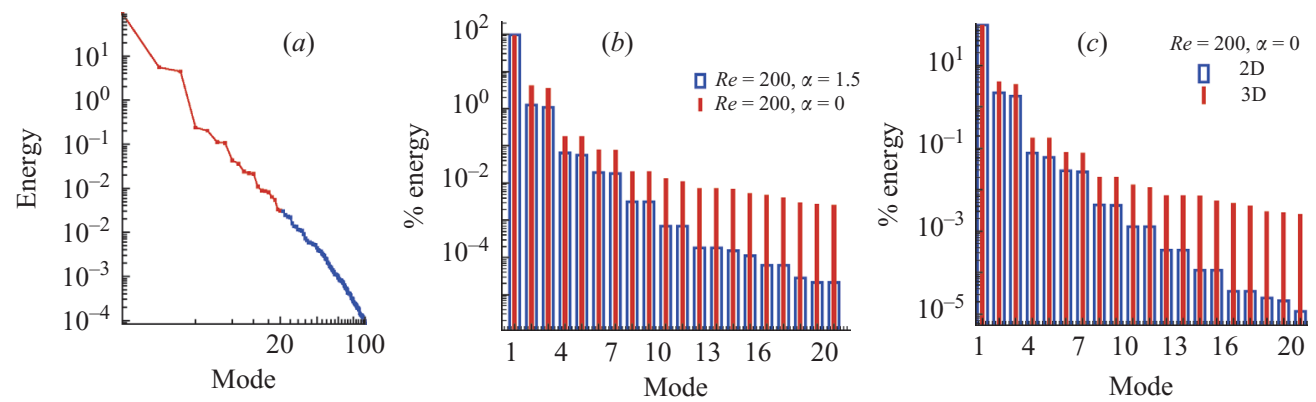

FIGURE 8. Energy diagram of the POD modes. (a) Energy contribution of the first 100 POD modes, $R e=300, \alpha=0.5$. (b) Rotation effect on the energy contribution of the first 20 modes. (c) Comparison of the first 20 POD modes in two- and three-dimensional simulations.

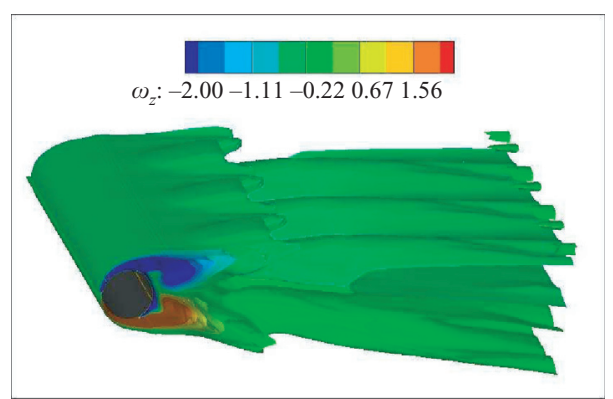

Mode 1

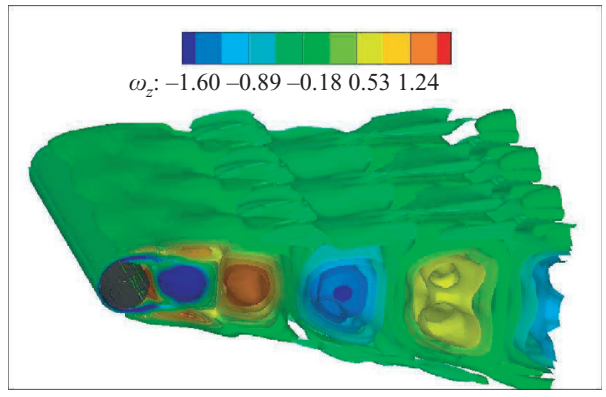

Mode 2

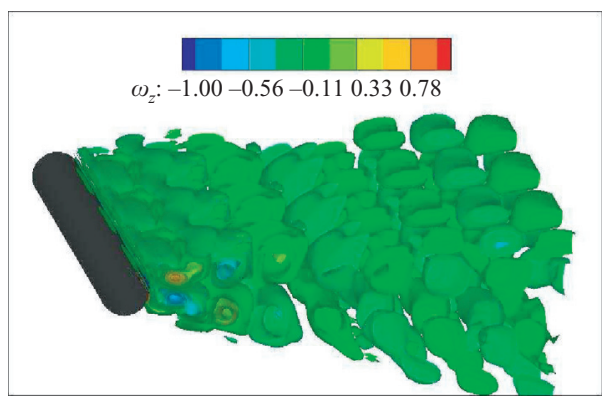

Mode 4

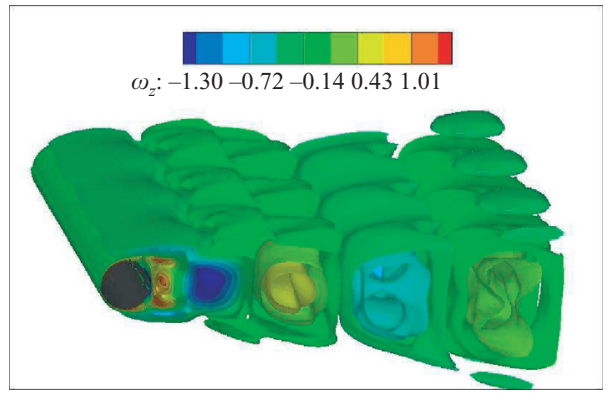

Mode 3

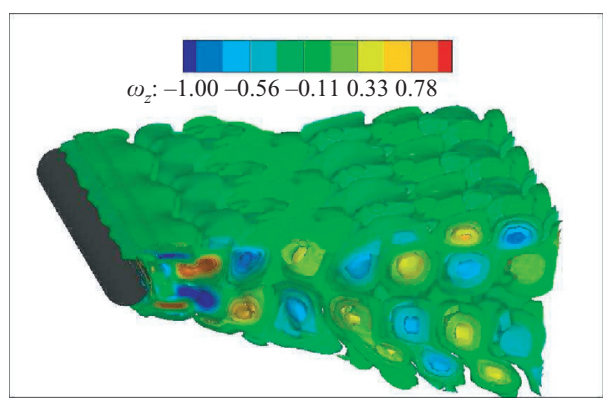

Mode 5

FIGURE 9. $\omega_{z}$ iso-contours of the first five three-dimensional POD modes, $R e=300, \alpha=0.5$. 

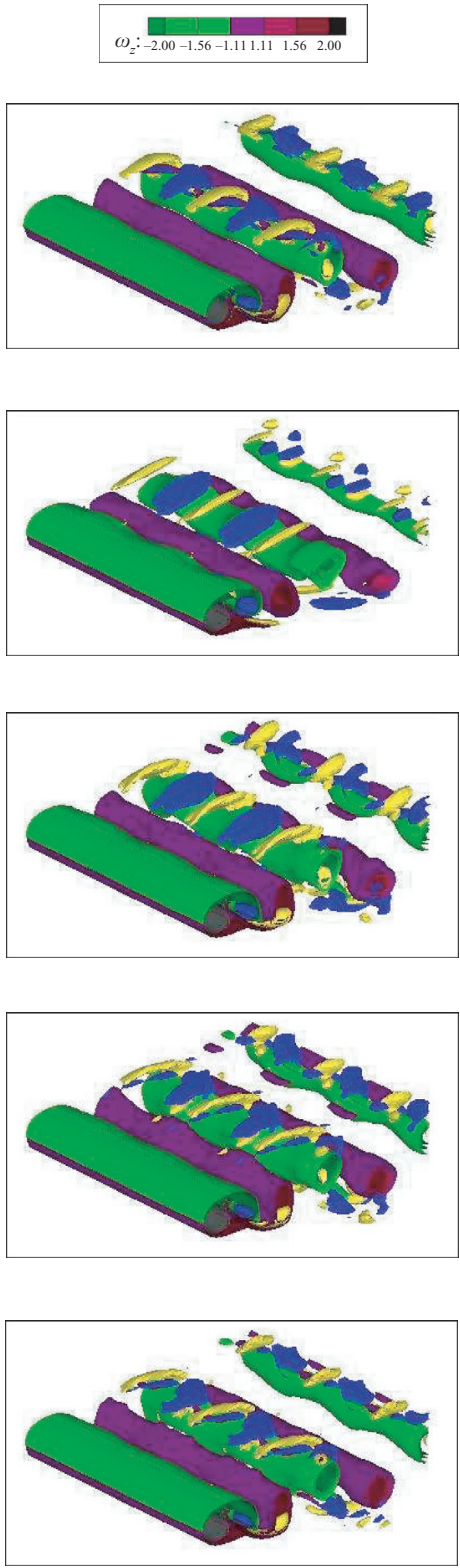

(a)
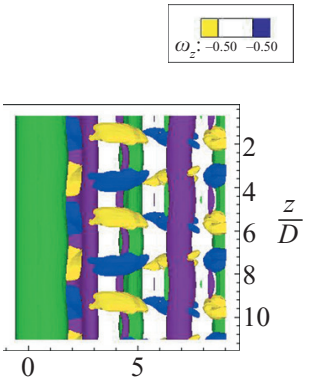

(b)

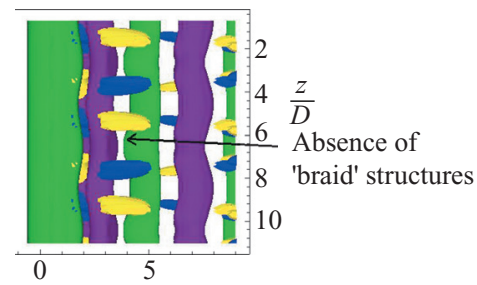

(c)

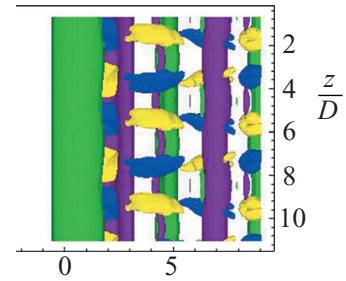

(d)

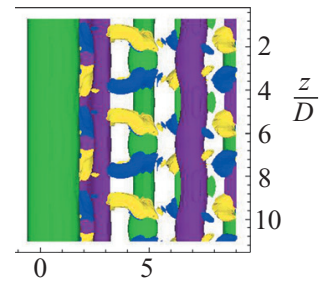

(e)

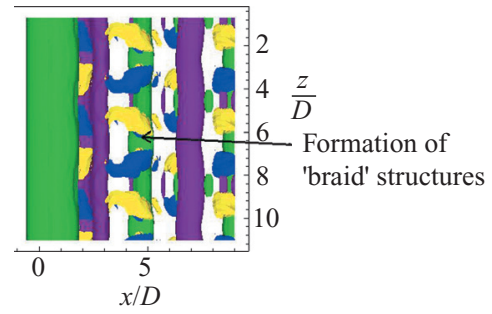

FIGURE 10. $\omega_{z}$ and $\omega_{x}$ iso-vorticity surface reconstructions for $\operatorname{Re}=300, \alpha=0.5$. (a) Instantaneous field, $(b)$ reconstruction with the first 3 modes, $(c) 7$ modes, $(d) 11$ modes, (e) 19 modes.

developed beyond $x / D=1 D$ and a two-vortex row is organized as with respect to the rear axis. The three-dimensional flow reconstruction is shown in figure 7. A 3-mode reconstruction does not provide a vortex pattern comparable with the instantaneous field. It can be seen that the flow pattern reconstructed with 11 and 19 modes compares quite well to the instantaneous one, especially concerning the spanwise undulation. This reconstruction also captures the modification of the strain axis of the 
coherent structures due to the rotation. The reconstruction with 19 modes shows the 'braid'-like three-dimensional pattern of the streamwise vortices more clearly than the 7-mode reconstruction. Therefore, to capture the secondary instability and to perform an efficient mode A reconstruction, an order of 15-19 modes is needed. It is recalled that the rotation introduces a global vorticity $\omega_{z}$ that is superimposed on the instantaneous vorticity distribution and strengthens the vorticity component in the $z$-direction. Therefore, the von-Kármán vortex rows become more stable to small spanwise perturbations, and as shown by the present DNS, they keep the wavelength of mode $\mathrm{A}$ at the present Reynolds number, 300.

\section{Conclusion}

The present study obtains two-dimensional transition steps in the flow around a rotating circular cylinder in good agreement with previous studies. The main objective is the analysis of the three-dimensional transition at low $R e$ and $\alpha$. A number of three-dimensional computations were carried out near the threshold of the bifurcation to the secondary instability. The amplification of the global instability is analysed by DNS and by a global oscillator model. It is shown that the rotation attenuates the secondary instability and increases the critical Reynolds number. This critical $R e$ has been evaluated as $R e_{c r}=219.8$, by the DNS approach and by the Landau model for $\alpha=0.5$. The analysis of the organized flow pattern was carried out by proper orthogonal decomposition. The three-dimensional flow reconstructions show that about 19 modes are sufficient to capture the secondary instability related to the spanwise undulation. This work has a significant implications for the understanding of the wall rotation effects that occur in a number of applications in rotating systems, as well as in flow control strategies using rotating cylinders.

We acknowledge valuable scientific discussions with G. Martinat, the collaboration of D. Faghani and A. Barthet in the POD approach, and the CPU allocations of the national computer centres CINES, IDRIS, CALMIP and GRIDMIP.

\section{REFERENCES}

Badr, H. M., Coutanceau, M., Dennis, S. C. R. \& Ménard, C. 1990 Unsteady flow past a rotating circular cylinder at Reynolds numbers $10^{3}$ and $10^{4}$. J. Fluid Mech. 220, 459-484.

Berkooz, G., Holmes, P. \& Lumley, J. 1993 The proper orthogonal decomposition in the analysis of turbulent flows. Annu. Rev. Fluid Mech. 25, 539-575.

Braza, M., Chassaing, P. \& Ha-Minh, H. 1986 Numerical study and physical analysis of the pressure and velocity fields in the near wake of a circular cylinder. J. Fluid Mech. 165, 79-130.

Braza, M., Faghani, D. \& Persillon, H. 2001 Successive stages and the role of natural vortex dislocations in three-dimensional wake transition. J. Fluid Mech. 439, 1-41.

Cliffe, K. A. \& Tavener, S. J. 2004 The effect of cylinder rotation and blockage ratio on the onset of the periodic flows. J. Fluid Mech. 501, 125-133.

Harlow, F. H. \& Welch, J. E. 1965 Numerical calculation of the time-dependent viscous incompressible flow of fluids with free surface. Phys. Fluids 8, 2182-2189.

Ingham, D. B. \& TANG, T. 1990 A numerical investigation into the steady flow past a rotating circular cylinder at low and intermediate Reynolds numbers. J. Comput. Phys. 87, 91-107.

JiN, G. \& BRAZA, M. 1993 A non-reflecting outlet boundary condition for incompressible unsteady Navier-Stokes calculations. J. Comput. Phys. 107, 239.

Kang, S., ChoI, H. \& LeE, S. 1999 Laminar flow past a rotating circular cylinder. Phys. Fluids 11, 3312-3321.

MittaL, S. 2004 Three-dimensional instabilities in flow past a rotating cylinder. J. Appl. Mech. 71, $89-95$. 
Mittal, S. \& Kumar, B. 2003 Flow past a rotating cylinder. J. Fluid Mech. 476, 303-334.

Peaceman, D. W. \& Rachford, J. R. 1955 The numerical solution of parabolic and elliptic differential equations. J. Soc. Indust. Appl. Maths. 3, 28.

Persillon, H. \& Braza, M. 1998 Physical analysis of the transition to turbulence in the wake of a circular cylinder by three-dimensional Navier-Stokes simulation. J. Fluid Mech. 365, 23-88.

PrandTL, L. 1925 Application of the "magnus effect" to the wind propulsion of ships. Die Naturwissenschaft 13, 93-108; transl. NACA-TM-367, June 1926.

Provansal, M., Mathis, C. \& Boyer, L. 1987 Bénard-Von Kàrmàn instability: transient and forced regimes. J. Fluid Mech. 182, 1-22.

Stojković, D., Breuer, M. \& Durst, F. 2002 Effect of high rotation rates on the laminar flow around a circular cylinder. Phys. Fluids 14, 3160-3178.

Stojković, D., Schön, P., Breuer, M. \& Durst, F. 2003 On the new vortex shedding mode past a rotating circular cylinder. Phys. Fluids 15, 1257-1260.

Tokumaru, P. T. \& Dimotakis, P. E. 1993 The lift of a cylinder executing rotary motions in a uniform flow. J. Fluid Mech. 255, 1-10.

Williamson, C. H. K. 1992 The natural and forced formation of splot-like vortex dislocations in the transition of a wake. J. Fluid Mech. 243, 393-441. 